\title{
RESEARCH
}

Open Access

\section{Maternal gut microbes shape the early-life assembly of gut microbiota in passerine chicks via nests}

\author{
Cheng-Yu Chen ${ }^{1}$, Chih-Kuan Chen ${ }^{1,2,3}$, Yi-Ying Chen ${ }^{1}$, Andrew Fang ${ }^{4}$, Grace Tzun-Wen Shaw ${ }^{1}$, \\ Chih-Ming Hung ${ }^{1 *}$ and Daryi Wang ${ }^{1 *}$ (i)
}

\begin{abstract}
Background: Knowledge is growing on how gut microbiota are established, but the effects of maternal symbiotic microbes throughout early microbial successions in birds remain elusive. In this study, we examined the contributions and transmission modes of maternal microbes into the neonatal microbiota of a passerine, the zebra finch (Taeniopygia guttata), based on fostering experiments.

Results: Using 165 rRNA amplicon sequencing, we found that zebra finch chicks raised by their biological or foster parents (the society finch Lonchura striata domestica) had gut microbial communities converging with those of the parents that reared them. Moreover, source-tracking models revealed high contribution of zebra finches' oral cavity/ crop microbiota to their chicks' early gut microbiota, which were largely replaced by the parental gut microbiota at later stages. The results suggest that oral feeding only affects the early stage of hatchling gut microbial development.

Conclusions: Our study indicates that passerine chicks mainly acquire symbionts through indirect maternal transmission-passive environmental uptake from nests that were smeared with the intestinal and cloacal microbes of parents that raised them. Gut microbial diversity was low in hand-reared chicks, emphasizing the importance of parental care in shaping the gut microbiota. In addition, several probiotics were found in chicks fostered by society finches, which are excellent foster parents for other finches in bird farms and hosts of brood parasitism by zebra finches in aviaries; this finding implies that avian species that can transfer probiotics to chicks may become selectively preferred hosts of brood parasitism in nature.
\end{abstract}

Keywords: Avian microbiota, Host-microbiome interactions, Maternal effects, Gut microbiota assembly, Maternal microbial transmission

\footnotetext{
* Correspondence: cmhung@gate.sinica.edu.tw; dywang@gate.sinica.edu.tw

${ }^{1}$ Biodiversity Research Center, Academia Sinica, Taipei 115201, Taiwan

Full list of author information is available at the end of the article
}

(c) The Author(s). 2020 Open Access This article is licensed under a Creative Commons Attribution 4.0 International License, which permits use, sharing, adaptation, distribution and reproduction in any medium or format, as long as you give appropriate credit to the original author(s) and the source, provide a link to the Creative Commons licence, and indicate if changes were made. The images or other third party material in this article are included in the article's Creative Commons licence, unless indicated otherwise in a credit line to the material. If material is not included in the article's Creative Commons licence and your intended use is not permitted by statutory regulation or exceeds the permitted use, you will need to obtain permission directly from the copyright holder. To view a copy of this licence, visit http://creativecommons.org/licenses/by/4.0/ The Creative Commons Public Domain Dedication waiver (http://creativecommons.org/publicdomain/zero/1.0/) applies to the data made available in this article, unless otherwise stated in a credit line to the data. 


\section{Background}

The gastrointestinal tract is now recognized as being largely sterile in newborn vertebrates [1], and is subsequently colonized by diverse bacterial taxa varying in abundance and functional traits [2,3]. These microbial communities, termed gut microbiota, may influence a wide range of metabolic, developmental, and physiological processes, affecting host health, fitness, and even behavior [4-6]. The transmission routes of symbiotic microbes-such as neonatal delivery, diet, environment, and parenting behavior-may shape the pool of potential colonists in gut microbiota [7-13]. On the other hand, inherited host-associated factors-such as genotype, sex, and immune status-may function as selective filters in the process of gut microbial community assembly [14-16].

Transmission routes have fundamental effects on microbial symbiont persistence and evolution [17]. Gut microbiota are transferred both from mother to offspring [8] and via other resources through social interactions, shared environment, and diet [11, 18-20]. Even though maternal transmission sensu stricto refers to direct transmission before birth, it is increasingly common for studies to also include indirect routes of maternal transmission [21], which now encompass any transfer of maternal symbionts to offspring during or after birth. In viviparous mammals, the initial colonists of the newborn gut come from maternal vaginal, fecal, and breast milk microbes; later on, a great degree of parental care may add diverse parental microbes, such as skin microbes, to newborns during their early stage of gut microbial community development [1, 22-24]. This process is crucial to the recruitment and establishment of neonatal microbiota and aid in defending against pathogens when the immune system is immature [25-28].

In birds, given that embryos develop in eggs-a closed and essentially sterile environment [29] - the chick gut may acquire microbes from eggshells [30, 31], maternal cloacal and fecal microbes smeared in the nest, or parents' oral microbes through feeding $[29,32]$. These transmission routes are especially important in altricial species, including passerines, because their chicks totally depend on parental care for survival [33]. Altricial chicks are typically raised in nests and fed with food that their parents catch or regurgitate. Although studies have suspected that eggshells carry the maternal microbes to chicks, no direct evidence for this has been found [31]. On the other hand, a recent study showed that the microbes of nests resembled those of internal (cloaca) and external (skin and feather) body sites in two lark species (Lullula arborea and Alauda arvensis) [34]. However, the roles of nests, eggshells, and oral feeding in mediating the early-life assembly of bird gut microbiota are still largely unclear.

A growing number of studies have investigated the transmission routes of early-life gut microbiota in natural populations of birds. In particular, avian brood parasitism, one of the most bizarre breeding strategies, provides a unique natural system for exploring transmission processes. Interspecific brood parasites lay their eggs in the nests of hosts, which incubate the parasitic eggs and raise the chicks. Therefore, parasitic chicks may acquire their gut microbiota from their biological mothers via direct transmission, foster parents via external (indirect) transmission, or both. Studies on parasitic cuckoos (Clamator glandarius) and their host magpies (Pica pica) suggest that genetic components are important in chick gut microbiota assembly, as magpie and cuckoo nestlings raised in the same nests have different gut microbial communities [35, 36]. In contrast, a study on parasitic cowbirds (Molothrus ater) suggested that geographic location and diet might exert strong effects on avian gut microbiota [37]. In addition, cross-fostering experiments in the field suggested that nesting environments may shape the cloacal bacterial assemblages in great tit (Parus major) and blue tit (Cyanistes caeruleus) nestlings; however, the lack of microbiota data on foster parents and nests prevented them from disentangling the confounding effects of the two components $[38,39]$. Overall, current research findings on the transmission routes and establishment of gut microbial communities in hatchlings are still controversial, as are possible changes in these routes over the course of chick development. Fostering experiments with careful designs in well-controlled conditions will be useful for addressing these issues.

The zebra finch (Taeniopygia guttata) is a wellestablished songbird model for animal behavior, neurobiology, and physiology [40, 41]. The society finch (Lonchura striata domestica) belongs to the same family of zebra finches, and has been bred over hundreds of years to be foster parents for other finch chicks, including zebra finches. Hence, a zebra finch-society finch fostering experiment in a well-controlled laboratory would be a good system to examine the complicated routes of gut microbiota transmissions. Interestingly, zebra finches were shown to lay their own eggs in society finch nests after their own nest was experimentally destroyed in aviaries; this behavior may provide a window into the evolution of brood parasitism in birds [42].

In this study, we aimed to examine the relative importance of genetic and maternal effects on the newborn gut microbiota. We further examined whether the maternal transmission of the chick gut microbiota mainly occurs through oral feeding (bacteria from parental oral cavities) or fecal contact in nests (bacteria from parental 
guts) across the different stages of chick development. We applied high-throughput sequencing to $16 \mathrm{~S}$ rRNA gene amplicons from the gut microbiome of zebra finch hatchlings raised by their biological parents, foster parents (i.e., society finches), and humans (Fig. 1), as well as from the oral cavity, crop, and gut microbiomes of the parents. Our results will also shed a new light on the gut microbiota assembly process during the initial phase of avian brood parasitism evolution.

\section{Methods}

\section{Finch fostering experiment and microbiota sample collection}

Pairs of adult finches used in this study were purchased from a breeder in Tainan, Taiwan (N 23 $14^{\prime}$ 09.7', E $\left.120^{\circ} 16^{\prime} 53.1^{\prime \prime}\right)$ and kept in a bird room at Biodiversity Research Center of Academia Sinica, Taipei, Taiwan, for at least 1 month before the experiments began. We maintained the bird room at $23-25^{\circ} \mathrm{C}, \sim 40 \%$ humidity, and a day-night cycle of $10 \mathrm{~h}: 14 \mathrm{~h}$ (as described in [41]). We housed each breeding pair in a wire cage, with dimensions of $45.5 \times 37.0 \times 41.5 \mathrm{~cm}$, and provided them with sterile coconut fibers as nesting materials. The seed component of the feed for all finches including adults and hatchlings comprised a mixture (by volume) of white millet (50\%) and canary seed (50\%), plus Niger seed (20 g per liter).

To investigate the contributions of maternal microbes to the early development of the gut microbiota in zebra finch hatchlings, we performed a fostering experiment on the zebra finch (ZF)-society finch (SF) system. Freshly laid eggs were collected from six pairs of adult ZF and randomly placed in the nests of SF or an external egg incubator (at $38{ }^{\circ} \mathrm{C}$ and $40 \%$ relative humidity; Octagon 20 Advance Semi, Brinsea Products Ltd, Standford, UK). After the egg collection, each zebra finch clutch consisted of 2-4 eggs, and the six ZF pairs continued to incubate the remaining eggs and then rear newborn chicks until a given post-hatching stage. To collect finch symbiotic microbiota, we anesthetized finches with isoflurane (Halocarbon Products Corporation, Peachtree Corners, GA, USA) prior to decapitation. After sacrificing the birds, we sampled the gut (including intestine and cloaca) contents of zebra finch hatchlings raised by their biological parents (ZF-reared chicks, cZF: $n=15$ ) or foster parents (SF-reared chicks, cSF: $n=21$ ) at four different developmental stages: 3, 6, 7 , and 10 days post-hatching (dph) (see details in Fig. 1).

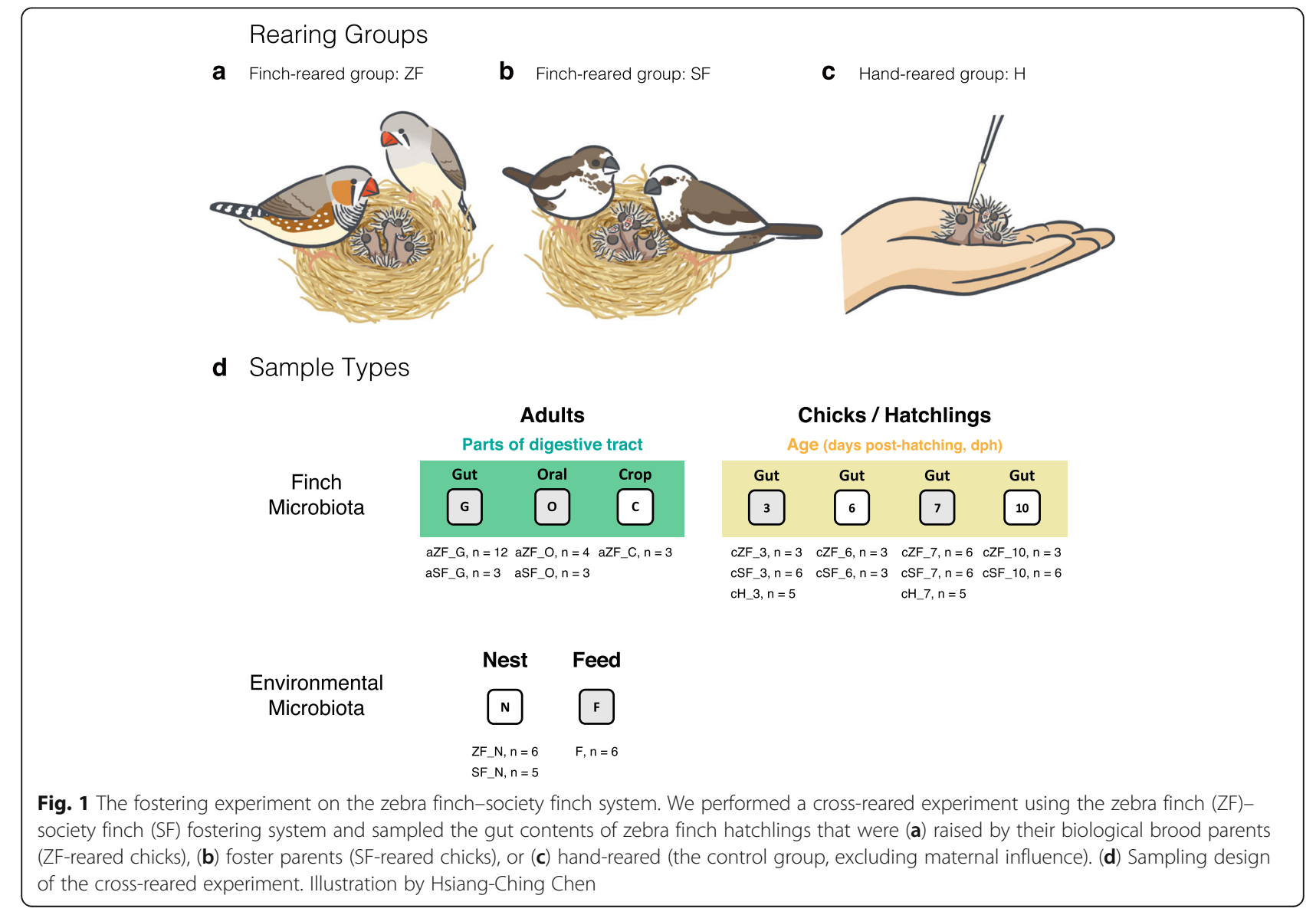


To create a control group that excluded maternal transmission via the nest environment and regurgitated food (but not eggshells), the incubator-hatching chicks ( $\mathrm{cH}: n$ $=10)$ were hand reared with homogenized feed (FastPrep-24 $4^{\text {Tu }}$ Homogenizer, MP Biomedicals) using a micropipette with sterile filtered pipette tips every $2-4 \mathrm{~h}$, sampled at 3 and $7 \mathrm{dph}$. We also sampled the oral swab (FLOQSwabs, Copan, Italy), crop, and gut contents of adult zebra finches (aZF: $n=12$ ) and society finches (aSF: $n=3$ ), as well as their nest materials (N: $n=11$ ) from the surface of nest bottoms, which the finches came in contact with most often, and feed samples (F: $n$ = 6). Comparing gut community dynamics in finchreared and control hatchlings allowed us to examine the influences of parental care and nesting environment on microbiota acquisition at that stage of hatchling development. All samples were stored at $-20{ }^{\circ} \mathrm{C}$ until DNA extraction.

\section{DNA extraction, amplification, and metagenomic sequencing}

Samples harvested after the birds were sacrificed were homogenized in $1.4 \mathrm{~mL}$ of lysis buffer (Buffer ASL, QIAamp DNA Stool Mini Kit) using FastPrep-24 Homogenizer (MP Biomedicals) in Precellys Lysing Kit CK14 tubes (Bertin Technologies, Montigny le Bretonneux, France). The bacterial DNA from homogenized finch (gut, crop, and oral) and environmental (nest materials and feed) samples were extracted using the QIAamp DNA Stool Mini Kit (QIAGEN, GmbH, Hilden, Germany) and quantified using a Qubit 2.0 Fluorometer (Invitrogen, Life Technologies, CA, USA).

The first two hypervariable regions (V1-V2) of the small subunit ribosomal RNA (16S rRNA) gene were amplified using universal eubacterial primers. The forward primer 27F (5'-AGAGTTTGATCMTGGCTCAG$\left.3^{\prime}\right)$ and reverse primer 355R (5'-GCTGCCTCCCGTAG GAGT-3') [43, 44] were fused with Illumina overhang adapters and specific 10-nt barcodes to allow multiple samples to be analyzed in parallel on a single picotiter plate. The pooled DNA was amplified with PCR (Taq DNA Polymerase $2 x$ Master Mix RED, Ampliqon, Odense $\mathrm{M}$, Denmark) under the following running conditions: initial denaturation for $3 \mathrm{~min}$ at $95^{\circ} \mathrm{C} ; 30$ cycles of $30 \mathrm{~s}$ at $95^{\circ} \mathrm{C}, 30 \mathrm{~s}$ at $55^{\circ} \mathrm{C}$, and $45 \mathrm{~s}$ at $72^{\circ} \mathrm{C}$; and a final elongation step for $10 \mathrm{~min}$ at $72^{\circ} \mathrm{C}$, modified from the Illumina standard protocol for $16 \mathrm{~S}$ metagenomic sequencing library preparation [45]. All PCR products were confirmed using $2 \%$ agarose gel electrophoresis and subsequently isolated from the gel and purified by NucleoSpin Gel and PCR Clean-up (Macherey Nagel, Düren, Germany). DNA concentrations of the clean PCR products were determined using a Quant-iT dsDNA HS assay kit and Qubit fluorometer (Invitrogen,
Carlsbad, CA, USA). The purified amplicons were further processed according to the Illumina standard protocol, and paired-end $2 \times 300 \mathrm{bp}$ sequencing was conducted on the MiSeq platform (Illumina, San Diego, CA, USA) with the reagent kit v3 at the NGS High Throughput Genomics Core Facility at Academia Sinica. All datasets were deposited into the Sequence Read Archive (SRA) database at NCBI under BioProject ID PRJNA609776. A full list of sample identifiers is provided in Supplementary Table S1 (Additional file 2).

\section{Sequence data processing}

The raw Illumina amplicon reads were demultiplexed and merged with the FLASH software [46], then quality filtered and analyzed using mothur v1.35.1 [47]. The criteria for filtering were minimum read length of $200 \mathrm{bp}$, maximum read length of $600 \mathrm{bp}$, minimum sequence quality score of 30 , maximum number of errors in the barcode of $1 \mathrm{bp}$, and maximum number of errors in the primer of $2 \mathrm{bp}$. Barcode and primer sequences were removed from the $5^{\prime}$ and $3^{\prime}$ ends, and chimeras were searched for and removed using the uchime_ref command in USEARCH [48]. After filtering and trimming, reads with an average length of $307 \mathrm{bp}$ among all samples were used for downstream analyses.

A UPARSE pipeline (usearch_global) [49] was used to cluster preprocessed reads into operational taxonomic units (OTUs) at 97\% similarity. The OTUs were further assigned into a taxonomic hierarchy based on the reference sequences from the Greengenes database (version 13.8.99) using mothur (classify.seqs) [50]. Sequences that were classified as chloroplast, mitochondria, eukaryota, or unknown were removed from the dataset (remove.lineage), as they likely represented ingested plant materials. Samples were rarefied to the minimum of 9557 sequences before being used for diversity analysis to avoid biases caused by uneven sequencing efforts.

\section{Statistical analysis}

We calculated the observed (OTU) richness and Shannon diversity (i.e., alpha diversity) index from rarefied data using mothur and applied analysis of variance (ANOVA) with Tukey-Kramer post hoc tests to analyze differences in microbiota diversity between rearing groups (i.e., hand-, SF-, and ZF-reared groups, including both adults and hatchlings) and sample types (i.e., guts, crops, oral cavities, nests, and feed). The adjusted $P$ values for pairwise Tukey-Kramer contrasts were reported from the default single-step method in the R package multcomp [51].

To assess the compositions of bacterial communities among groups or sample types (i.e., beta diversity), we used Bray-Curtis dissimilarities and weighted UniFrac [52] to evaluate phylogenetic similarities. The non- 
metric multidimensional scaling (NMDS) ordination approach [53] based on Bray-Curtis dissimilarities from the OTU-level table was conducted using the $\mathrm{R}$ package vegan [54] to perform a parallel comparison. Apparent changes in community structure were tested using analysis of molecular variance (AMOVA) [55] in mothur.

The average relative abundance of the most prevalent bacterial families (abundance $>0.5 \%$ ) was estimated for each sample type and plotted for samples from the three rearing groups and feeds. To further measure the specificity of a bacterial taxon (at the family level) to a given sample type, we determined its indicator value (IndVal) index, which considers the relative abundance of a taxon in a given community and its relative frequency of occurrence across all communities. The IndVal index quantified the specificity of taxa from those found in only a single community to those found across all communities. Bubble plots of relative taxonomic abundances and IndVal were generated using the R package ggplot to assess the impacts of microbiota on adult digestive systems, feed, and nest materials on those in chick guts.

To identify significantly different taxa among hatchlings from hand-, SF-, and ZF-reared groups, the linear discriminant analysis (LDA) effect size (LEfSe) was performed using the online Huttenhower Galaxy server (http://huttenhower.sph.harvard.edu/galaxy/). In this analysis, LEfSe determined the active bacterial taxa (from the phylum to genus levels) in the finch gut microbiome based on changes in OTU abundance to explain differences among development stages and rearing groups. We then used SourceTracker2 [56], a Bayesian community-level microbial source-tracking tool, to estimate the proportion of sequences in the hatchling gut microbiota that originated from their biological parent, foster parent, or environmental communities. SourceTracker2 was run with default parameters using nonrarefied data; each hatchling gut microbial community was designated as a sink, and all other finches and environmental sample types were designated as sources.

\section{Results}

\section{Richness and diversity of finch and environmental microbiota}

Our 16S rRNA sequencing effort produced 6,265,933 quality filtered reads clustered into 2,553 OTUs. Sequence coverage ranged from 9557 to 149,280 reads per sample. The coverage ranges of different sample types were narrower (feed: 9557-39,711, nest: 48,572-130,324, oral: $77,726-131,807$, crop: $50,730-122,496$, and gut: 10 , 800-149,280; see rarefaction curves in Additional file 1: Figure S1 and detailed information in Additional file 2: Table S1) than that of the total range.

We found that the finch and environmental microbiota varied across different sample types. The environmental samples (nest and feed) harbored higher OTU richness and more diverse microbiota than finch samples from the different parts of adult digestive tracts (oral cavities, crops, and guts) and from the guts of hatchlings at different days post-hatching in both finch-reared groups (Fig. 2). In finch gut microbiota, observed OTU richness was not significantly different between the two finch-reared groups, including parents and hatchings (SF-ZF: ANOVA, $F_{1,49}=$ $0.048, P=0.83$ ), or among hatchlings raised by human and finch parents (cH-cF: $F_{1,44}=0.153, P=0.698$ ) (Fig. 2a). Nevertheless, the finch-reared hatchlings had significantly higher alpha diversity (Shannon index) levels in their gut microbiota than those of hand-raised ones $(\mathrm{cH}-$ cF: $F_{1,44}=11.920, P=0.001$ ).

Age had a major impact on finch gut microbial diversity, as we observed modest reductions in OTU richness (chicks-adults: $F_{1,59}=5.224, P=0.026$ ) and strong decreases in Shannon index (chicks-adults: $F_{1,59}=9.832, P$ $=0.002)$ from hatchings to adults. Among finch-reared hatchlings, the Shannon diversity of chicks raised by society finches continued to decrease over the first 10 days after hatching, whereas those of ZF-reared chicks did not show such an age-associated decline (Fig. 2b).

\section{Early-life finch gut microbial community structure}

We next sought to examine how the hatchling gut microbial communities varied across different rearing groups at 3, 6, 7, and 10 days post-hatching (dph) (Fig. 3 and Additional file 1: Figure S2). The SF- and ZF-reared chicks did not show distinct gut microbial community structures at any age (Fig. 3a). On the other hand, the gut microbiota in the hand-raised chicks were somewhat clustered and separated from those of finch-reared chicks (cH-cF: AMOVA, $F s_{1,44}=7.019, P<0.001$ ), while the feed microbiota was the closest bacterial community to the former (Fig. 3a). Pairwise Bray-Curtis dissimilarity comparisons between communities (beta diversity) further corroborated these observations. The beta diversities of microbiota between finch-reared chicks (cSF and cZF) and samples obtained from their parents and nest environment were significantly lower than those between hand-reared chicks $(\mathrm{cH})$ and other samples (Additional file 1: Figure S3, dashed line versus solid line). In the finch-reared hatchlings, adult oral and crop communities were significantly different from 10-dph chick gut communities (aFO-cF10: $F s_{1,14}=$ 4.422, $P<0.001$; aFC-cF10: $\left.F s_{1,10}=2.835, P=0.005\right)$, but were less different from the 3-dph ones (aFO-cF3: $F s_{1,14}=$ 2.903, $P=0.003$; aFC-cF3: $F s_{1,10}=1.995, P=0.06$ ) (Fig. 3); in contrast, adult gut communities were more different from 3-dph chick gut communities (aFG-cF3: $F s_{1,22}=$ 9.088, $P<0.001)$ than from 10 -dph ones (aFG-cF10: $F s_{1,22}$ $=6.445, P=0.004)$ (Fig. 3a, b).

Of the 30 total identified bacterial phyla, six dominated the finch gut microbiota (average cumulative abundance $=$ 

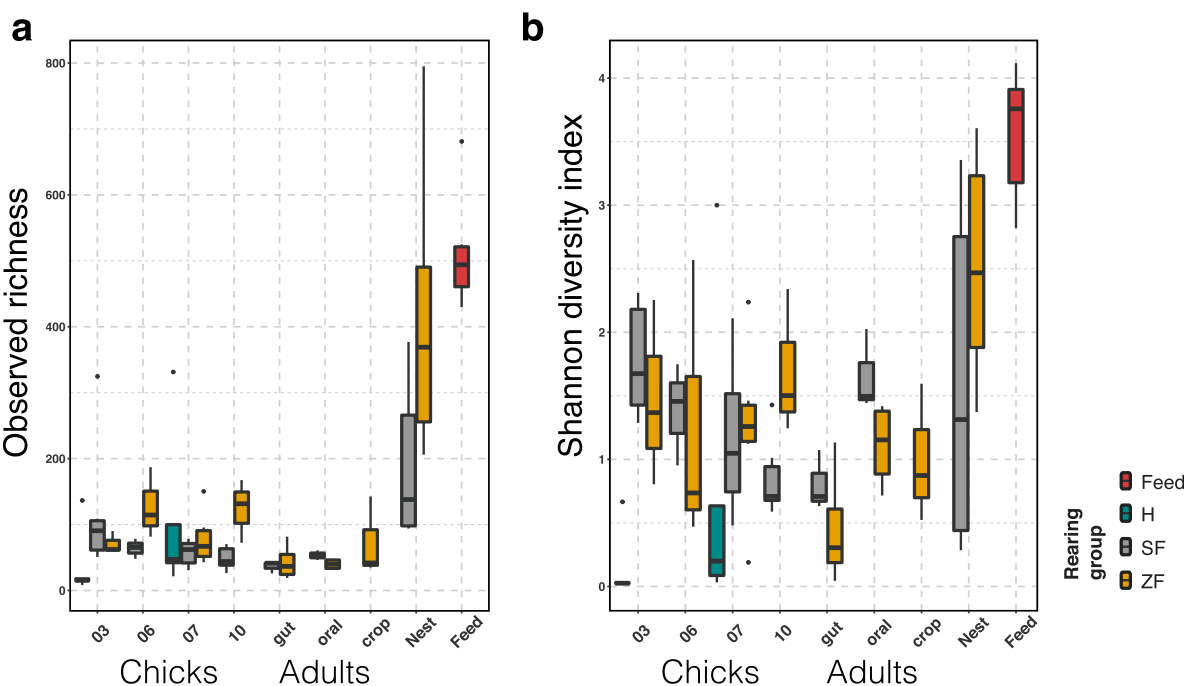

Fig. 2 Alpha diversity across sample types of hand- (H), society finch- (SF), and zebra finch (ZF)-reared groups. (a) The observed richness and (b) Shannon diversity index of microbial communities in samples from the hatchling gut (at 3, 6, 7, 10 days post-hatching, dph), adult digestive tract (gut, oral, crop), and environment (nest materials and feed). The hand-reared group included only hatchling gut samples at 3 and $7 \mathrm{dph}$

99.5\%), including the phyla shown to be prevalent in avian gut communities-Firmicutes, Proteobacteria, Actinobacteria, and Bacteroidetes-in other studies [57] (Fig. 4a). Firmicutes comprised the most dominant phylum among the hatchling gut microbiota, except in the 3-dph handreared chicks. However, within these Firmicutesdominated communities, the three rearing groups possessed different distributions at the family level (Fig. 4b and Additional file 1: Figure S4). Lactobacillaceae and Enterococcaceae were prevalent in the finch-reared hatchlings. SF-reared chicks harbored on average the largest fraction of Lactobacillaceae, the proportion of which increased over the growth of hatchlings (average abundance from $16.2 \%$ at $3 \mathrm{dph}$ to $73.4 \%$ at $10 \mathrm{dph}$ ), opposite to that of Enterococcaceae (average abundance from $38.2 \%$ at 3 dph to $22.2 \%$ at $10 \mathrm{dph}$ ); ZF-reared chicks showed a relatively stable pattern of age variation during growth. Noteworthy, the predominant members of the gut microbiota in adults-Lactobacillaceae for the society finch (average abundance $=87.3 \%$ ) and Campylobacteraceae for the zebra finch (average abundance $=73.0 \%$ )-were also prevalent in the hatchlings they raised and their nest environments. In contrast, the hand-raised chicks had few signature taxa except Enterobacteriaceae and Enterococcaceae, which were predominant at 3 and $7 \mathrm{dph}$, respectively (average abundance $=97.3 \%$ and $76.7 \%$, respectively), consistent with the lowest bacterial diversities among all experiment groups (Fig. 2).
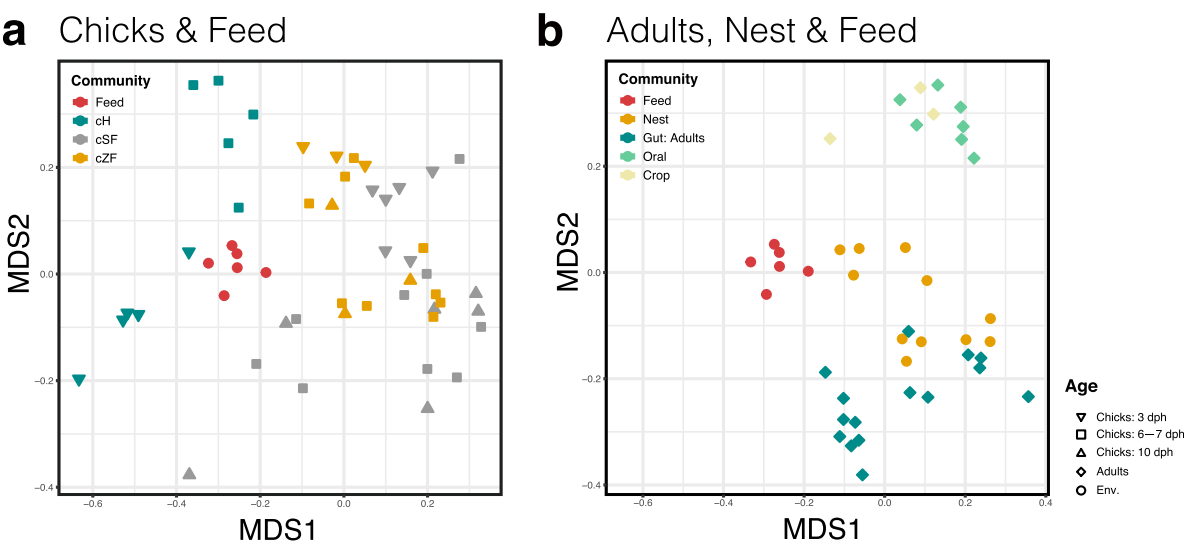

Fig. 3 Microbial community structures across finch and environmental samples. Non-metric multidimensional scaling (NMDS) ordination on BrayCurtis distances among sample types was plotted based on OTU abundances in (a) chick, (b) adult, and environmental (Env.: nest and feed) samples. CH, CSF, and CZF: ZF chick gut microbiota from hand-, society finch-, and zebra finch-reared groups 
a Phylum

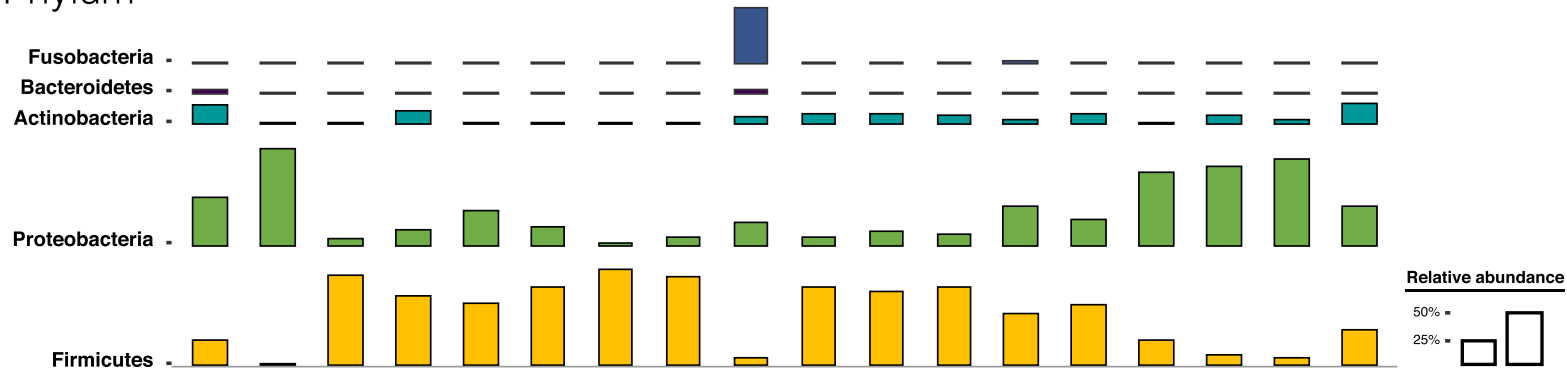

\section{b Family}

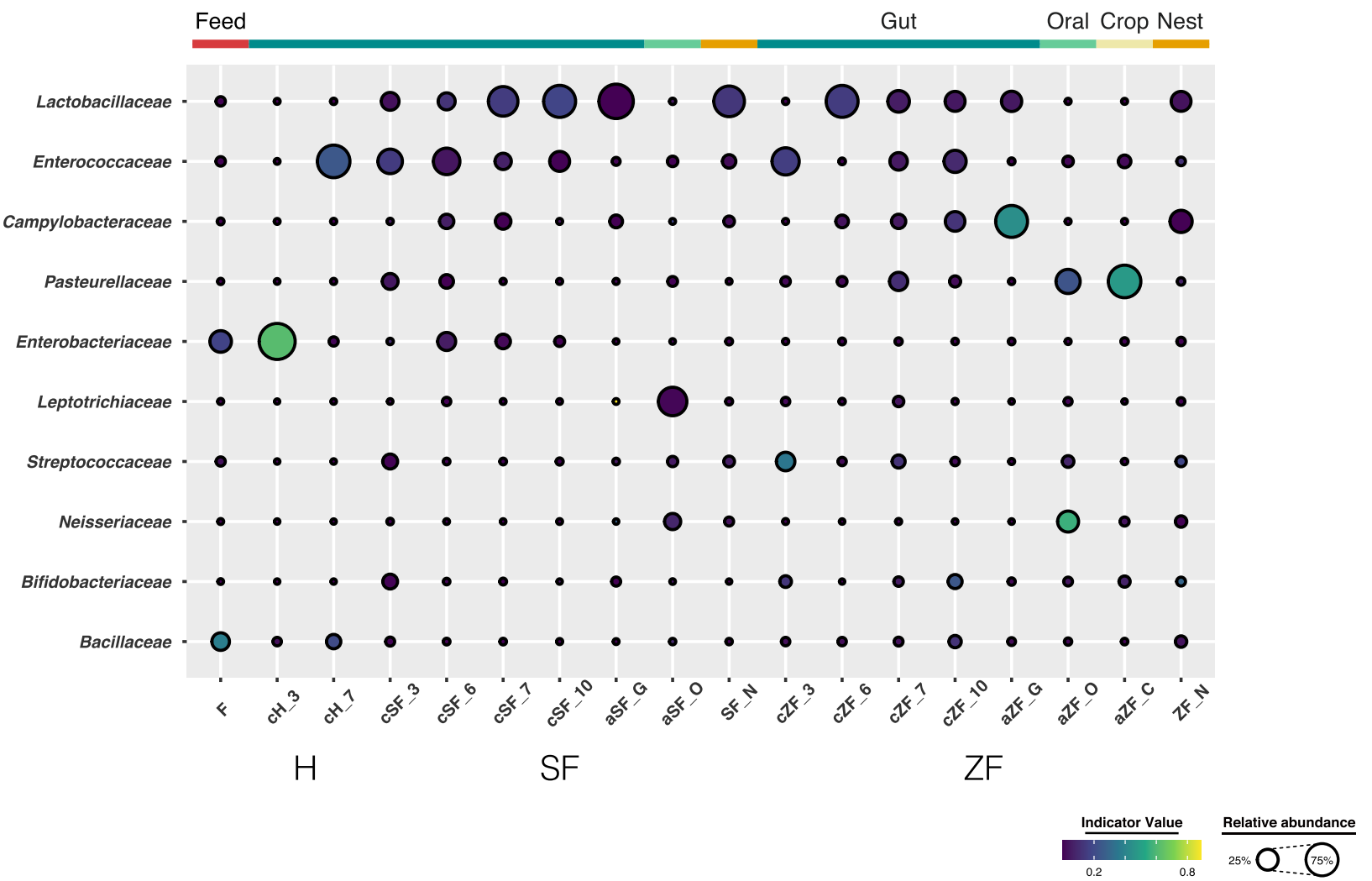

Fig. 4 Microbial compositions of the finch and environmental microbiota. The average relative abundances of the most prevalent bacterial (a) phyla (bar length) and (b) families (circle size) in each sample type (indicated as above bubble plot) plotted for samples from the three rearing groups and feed. The indicator value index (shading of circle color) represents the strength of association between a taxon and a given sample type, with larger values indicating greater specificity

To further measure the specificity of a taxon to a given sample type, we determined its indicator value (IndVal) index. For instance, in the hand-reared group, Enterobacteriaceae was specific to the chicks at $3 \mathrm{dph}$ (IndVal $=0.616$; Fig. 4b), whereas Bacillaceae had a relatively higher specific index in the 7-dph chicks (IndVal = 0.205 ) and feed microbial communities (IndVal $=0.375$ ). In addition, the oral cavity and crop of zebra finch adults were characterized by Pasteurellaceae (oral: 36.9\%, IndVal $=0.230$; crop: $76.6 \%$, IndVal $=0.477$ ), whereas their gut was characterized by Campylobacteraceae
(IndVal $=0.436$ ); the above two bacterial phyla also occurred in the gut microbiota of the ZF-reared chicks.

Influence of the maternal microbiota on the early stages of finch gut microbial successions

To determine the association between early gut microbial successions and parental care, linear discriminant analysis effect size (LEfSe) analysis [58] was performed to identify representative taxa in given communities. An appreciable resemblance was detected between the gut microbiota of the hatchlings and their feeding parents 
(Fig. 5 and Additional file 1: Figure S5). Consistent with the microbial composition results (Fig. 4b), the LEfSe results showed that the adult gut microbiota of society finches enriched in Lactobacillaceae, Bifidobacteriaceae, and Leptotrichiaceae, while that of zebra finches enriched in Bacillaceae and Campylobacteraceae (at the family level; Fig. 5d). In the SF-reared chicks, Bifidobacteriaceae and Neisseriaceae were dominant at $3 \mathrm{dph}$ (Fig. 5a). By 7-10 dph, had Lactobacillaceae increased appreciably and become the dominant bacterial family (Fig. 5b, c). In contrast, Streptococcaceae, Neisseriaceae, and Pasteurellaceae were relatively abundant in the 7-dph ZF-reared chicks, and then Bacillaceae and Pasteurellaceae became dominant at $10 \mathrm{dph}$ (Fig. $5 \mathrm{c}$ ). In the hand- raised group that was designed to eliminate (external) maternal transmission, only Enterobacteriaceae and Enterococcaceae were predominant in chicks at 3 and 7 dph, respectively (Fig. 5a, b). Thus, the LEfSe results of taxa at the family level further demonstrated that maternal gut microbes were the substantial contributor to early-life successions in the finch gut community.

\section{Predictive source tracking of hatchling gut communities}

We finally used Bayesian community-level source tracking [56] to investigate how maternal or environmental sources contributed to the gut community assembly of hatchlings. The SourceTracker model also revealed the major role of maternal gut microbes in shaping finch-reared chick gut

\section{a Chicks: 3 dph}

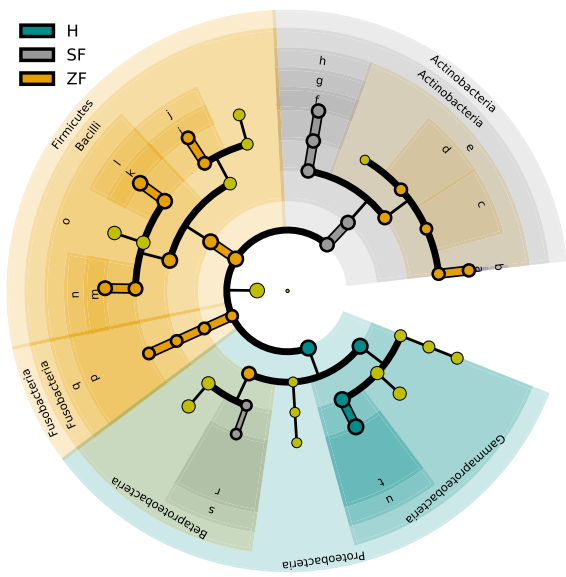

C Chicks: $10 \mathrm{dph}$

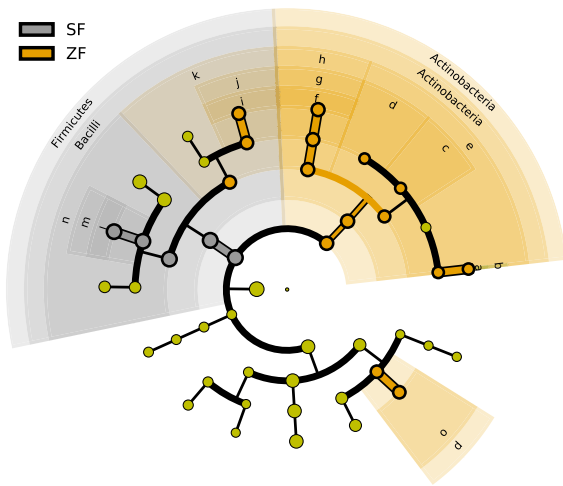

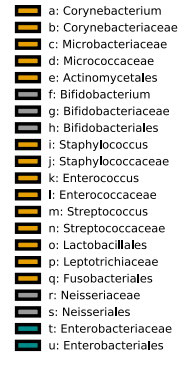

b Chicks: $7 \mathrm{dph}$

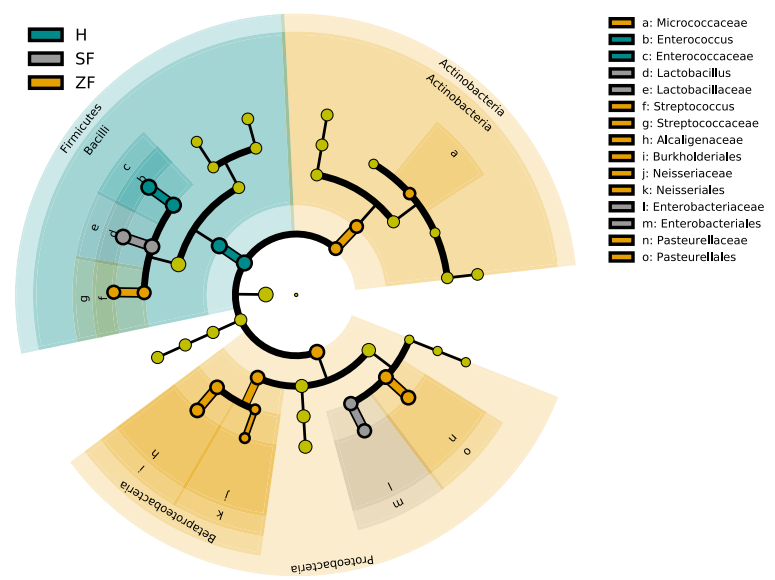

Adults

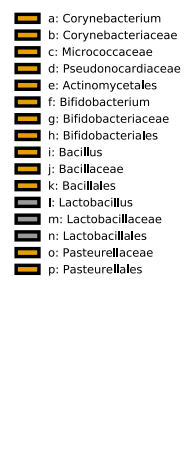

a: Pseudonocardiacea 它: Bifidobacterium d: ififidobacteriales 吕 $\begin{gathered}\text { e: Bacillus } \\ \text { f: Bacillacea }\end{gathered}$ 9: Lactobacillus n: Lactobacillacea 官 : Lactobaciliales 官: Campylobacteraceae 号: i: Leptotrichiaceace

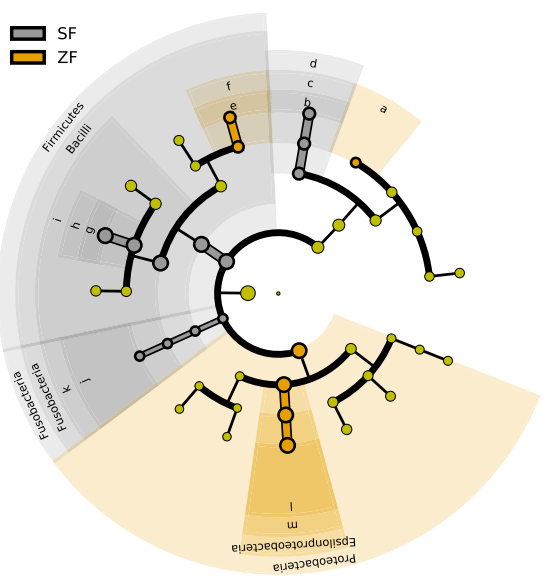

Fig. 5 The active bacteria in the finch gut microbiota, determined by LEfSe among three rearing groups. The gut microbiota of (a) 3-, (b) 7-, (c) 10-dph chicks, and (d) adults from hand (H)- (green), society finch (SF)- (gray), and zebra finch (ZF)-reared group (gamboge yellow) were compared. Colors indicate taxa or branches of the tree, which more significantly represent certain groups than others, except that gray yellow circles indicate taxa with non-significant differences 
microbial communities, especially at $10 \mathrm{dph}$ (Fig. 6b, c). On average, $71 \%$ of the SF-reared chick gut microbiota were from maternal gut sources, $3 \%$ from nesting environmental sources, less than $1 \%$ from feed, and $24 \%$ from unknown sources; on average, $24 \%$ of the ZF-reared chick gut microbes were from maternal gut sources, $12 \%$ from maternal crop sources, $7 \%$ from the nest, and 55\% from unknown sources. The results were consistent with the patterns derived from the taxonomy and linear analyses (Figs. 3, 4, 5, and Additional file 1: Figure S2). Despite a noticeable level of compositional mismatch between ZFreared chick gut microbiota and considered source communities, SourceTracker identified maternal oral and crop communities as dominant sources of chick gut communities from 3 to $7 \mathrm{dph}$. In contrast, maternal oral and crop communities contributed little to the gut communities of SF-reared chicks at any stage. Using SourceTracker to estimate the origin of hand-reared chick gut microbiota, we found to our surprise little evidence that feed microbes colonized hatchling guts, which had microbiota with largely unknown sources (Fig. 6a; see the "Discussion" section for details).

\section{Discussion}

Although still scarce, several studies in recent years have investigated the early establishment of the gut microbiota in birds such as chickens [32], ostriches [59], and shorebirds [29]. Avian brood parasitism and fostering experiments in the field [36, 37, 39] have been used to evaluate the maternal and environmental effects on the

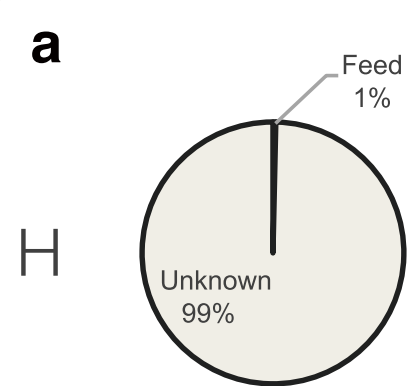

b
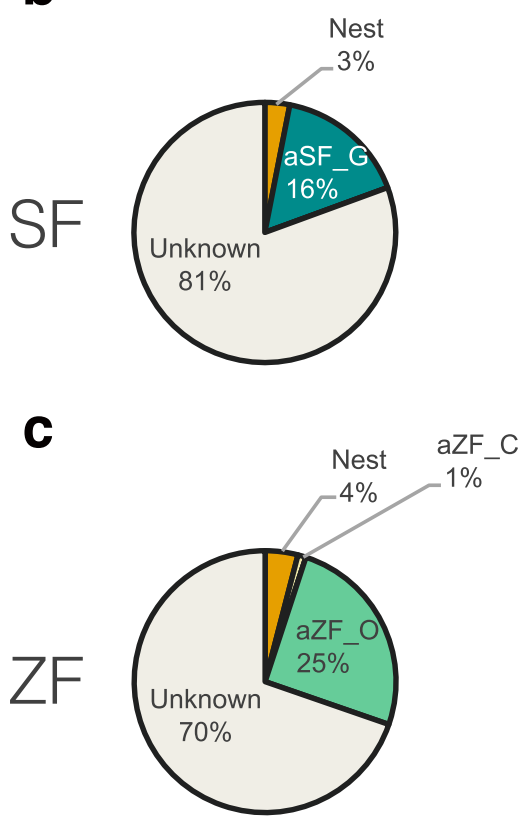

$3 \mathrm{dph}$
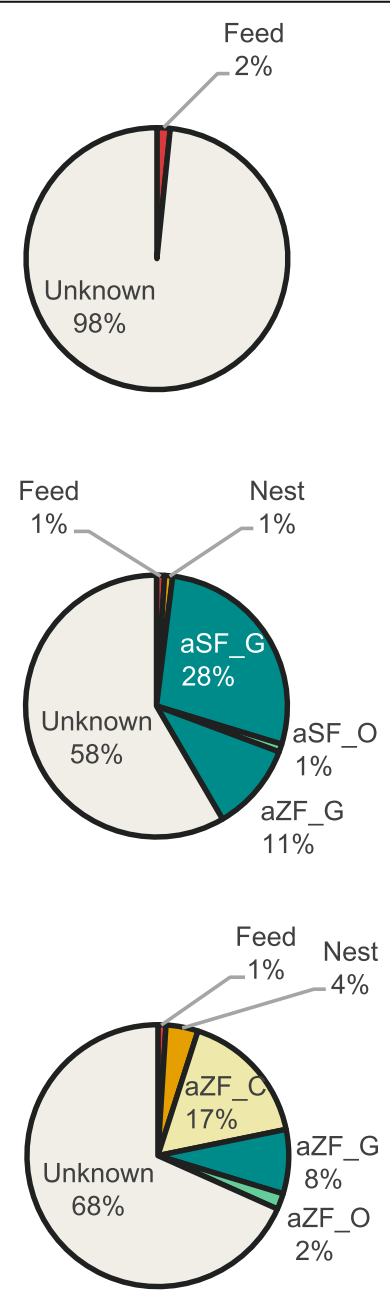

$6-7 \mathrm{dph}$

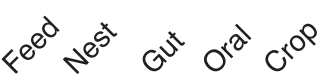

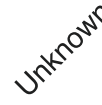

Env.

Maternal
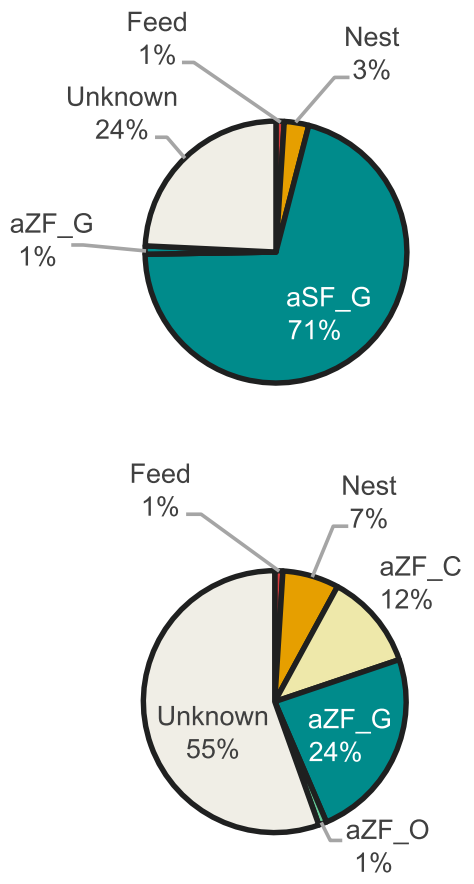

$10 \mathrm{dph}$

Fig. 6 Community-level source-tracking models for the maternal and environmental sources of early gut community assembly. The predicted proportions of sequences in the hatchling gut microbiota of (a) hand (H)-, (b) society finch (SF)-, and (c) zebra finch (ZF)-reared chicks at different days after hatching $(3,6-7$, and $10 \mathrm{dph})$ that originated from their biological parents, foster parents, or environmental communities 
gut microbial assembly of newborns. However, previous studies based on natural populations often show conflicting results, probably due to the confounding effects of parental (feeding behaviors) and environmental (available food or habitats) microbial transmission. Here, we studied the zebra finch-society finch fostering system in a well-controlled environment as an alternative strategy to further determine microbial transmission routes across several stages of hatchling ontogeny. Our results demonstrate that the gut microbial community of newborn zebra finches resembles that of the adult finches that reared them. That is, chick guts obtain and accumulate parental gut microbes after hatching, and genetics do not play an important role in this process. Our study also provides the first evidence that avian hatchling gut microbiota are mainly shaped by the transmission of parental gut microbiota via nests, especially at later stages of chick development.

\section{Nesting environment as a crucial route for maternal transmission to shape the hatchling gut microbial community}

Chicks generally harbor more diverse gut microbiota than their parents, and the differences decrease as they grow. In the SF-reared group, the alpha diversity and richness of hatchling gut microbiota are the highest in neonatals (3-dph chicks), and subsequently decrease during maturation. Our results are in contrast to the pattern of mammal gut microbial establishment; mammal gut microbial diversity increases progressively beginning with the initial colonization during delivery because parental care supplements the young with parental microbes $[1,60]$. However, several studies on wild bird species [29, 39, 61, 62] congruent with our results suggest that newborns already harbor diverse gut microbiota owing to the rapid colonization of gut microbes shortly after hatching. The high microbial diversity of hatchling gut communities might come from not only the biological mother (via the eggshell), but also feeding parents through direct contact during food provisioning (via maternal oral cavities or crops) or passive exposure to the nesting environment (via maternal feces). We further discuss the relative importance of these three transmission routes using our analytical results.

We found little evidence that the biological mother transmits her gut microbiota via eggshells. A previous study found that eggshells have bacterial communities strongly resembling those of the maternal skin, feather, and nest material, and therefore may act as a transgenerational carrier of maternal microbiota to the hatching offspring [31]. However, the hatchlings in our control group, for which possible maternal transmission is confined to eggshells, did not harbor gut microbiota resembling those of their biological mothers. Instead, the gut microbiota of 3-dph hand-reared hatchlings had low diversity and richness, and only shared a few bacteria with its feed. The results suggest a trivial role of eggshells in transferring maternal symbiotic microbes for the initial establishment of the avian gut microbiota. The low gut microbial diversity of hand-reared chicks also emphasizes the importance of parental feeding and/ or nesting environment in gut microbiota establishment.

Maternal transmission via regurgitated food is relatively important at the early stage of zebra finch development, and its impact diminishes as chicks grow. This argument is supported by the results that zebra finch parents' oral cavity/crop microbiota strongly contributed to chicks' early gut microbiota, which were largely replaced by parental gut microbiota at later development stages (Fig. 6). However, the gut microbiota of fostered chicks was mainly contributed by the gut microbiota of society finches throughout their development. Thus, maternal transmission via regurgitated food is only observed in chicks reared by their biological parents, not in those reared by foster parents. The results imply that inter-species differences in immune systems or physiological factors may determine the role of oral feeding on early gut microbiota establishment in chicks.

Most importantly, our data suggest that the gut microbiota of hatchlings raised by finches is assembled mainly through maternal transmission via the nesting environment. We found that the nest microbiota significantly resembled the microbial composition of adult guts, and as hatchlings stayed in nests longer their gut microbiota converged with that of the parents that reared them. Nests often accumulate parental feces, which likely contain bacteria from their guts. Hence, our results suggest that the nesting environment may function as a transgenerational carrier of maternal gut microbes-a route for maternal transmission that has not been directly tested before $[38,39]$.

\section{Maternal effect on gut microbiota assembly during hatchling development}

Alongside the decrease in microbial diversity over hatchling development, the contributions of the maternal gut community substantially increased in finchreared groups. Thus, maternal gut symbionts are the major contributors to the establishment of avian gut microbiota. The predicted active and dominant bacterial families in the adult gut-Campylobacteraceae in the zebra finch and Lactobacillaceae in the society finchalso became prevalent in the hatchlings they raised at $10 \mathrm{dph}$. In the ZF-reared group, the major shift in the hatchling gut microbial composition was from Enterococcaceae at $3 \mathrm{dph}$ to Campylobacteraceae at $10 \mathrm{dph}$. Campylobacter and related genera in the family Campylobacteraceae are oral and intestinal commensals of 
vertebrates [63], and may be one of the important sources of mortality and morbidity in wild and domestic animal populations [64]. However, Campylobacter spp. are generally accepted to be nonpathogenic in avian hosts [65]; they were consistently isolated from the guts of zebra finches that were outwardly healthy [66]. In the SF-reared group, the major bacterial family changed from Bifidobacteriaceae at $3 \mathrm{dph}$ to Lactobacillaceae at $10 \mathrm{dph}$. Many gut symbiotic microbes in these two families are considered as probiotics that play an important role in nutrition, growth, and protection from infection $[4,67,68]$. Both microbial families are the LEfSe-predicted active bacteria in adult society finches. This finding supports a common opinion in pet bird farms that the society finches are excellent foster parents for other finches, probably because they can maintain a healthy gut microbiota in hatchlings during early-life development.

In contrast, several candidate probiotics, such as Lactobacillaceae and Bifidobacteriaceae, were not well established in the gut communities of the hand-reared hatchlings. In fact, the gut microbiota of these hatchlings had a low diversity and richness, and shared the predominant bacterial family Enterobacteriaceae with their feed only at $3 \mathrm{dph}$. Enterobacteriaceae may reflect the influence of the Proteobacteria-rich microbiota, which is associated with xylanase for cellulolytic pre-digestion of feed in birds [57]. The hand-raised hatchlings at $7 \mathrm{dph}$ harbored a gut bacterial population dominated only by Enterococcaceae, which is one of the prevalent avian intestinal microbes $[57,65,69,70]$ and was also observed in the hatchling gut communities from the finch-reared groups. In the hand-reared group, the feed microbial community seemed to be the major and perhaps only contributor according to the taxonomic composition, although our source tracking results failed to provide statistical support due to a low number of reads in this group. It is possible that feed microbes are important colonists for the hatchlings of all groups, but are disproportionately excluded by the host immune system or gut environment. The reason for the large unknown source in the tracking results is that this analysis does not consider all source-sink scenarios, nor does our study include other potential environmental sources (e.g., the incubator box for the hand-reared group). Nevertheless, our results highlight the importance of parental care in shaping the gut microbiota in birds. Such maternal effects could be critical to the health of chicks.

\section{Conclusion}

The gut microbiota is shaped by multiple factors, and the relative contributions of host genetics and environments remain uncertain. In recent years, the environment has come to be considered far more important than host genetic relationships in determining host gut microbiota [11]. Among the environmental factors, diet has emerged as a pivotal determinant of gut microbiota community structure and function [71, 72]. On the other hand, growing evidence points out that avian gut microbiota are likely determined by habitat locations or the nests in which chicks are reared [31, 34, 37, 39, 73, 74]. In natural populations, the effects of habitat locations, nests, and diet on shaping the microbiota of young chicks are inseparable. Thus, in this study, we controlled the genetics and diet in simplified environmental conditions and used fostering experiments to estimate the maternal effects on the hatchling gut microbiota from 3 to $10 \mathrm{dph}$. Our results suggest little impact of genetics and diet on the hatchling gut microbiota. We found that young hatchlings initially had gut microbiota distinct from those of adults, and undergo a transition period until reaching a stable, mature adult-like microbial community. Among all the maternal symbionts we collected (from oral cavities, crops, and guts), maternal gut microbes contributed the most to the microbial community of hatchlings, especially toward their late development stages. Noteworthy, our study identified nests, which are often smeared by parental cloacal and fecal bacteria, as a crucial route for maternal transmission on early gut microbial assembly, supporting a notion of "Like mother like nest" [75]. Furthermore, many candidate probiotics were found in finch-reared hatchlings, especially those fostered by society finches, illuminating the positive effects of parental care on gut microbiota assembly during hatchling development. It also implies that wild avian species that can transfer probiotics to chicks may become selectively preferred hosts for parasitic birds like cuckoos and cowbirds. This hypothetical relationship warrants further study.

\section{Supplementary information}

Supplementary information accompanies this paper at https://doi.org/10. 1186/s40168-020-00896-9.

\footnotetext{
Additional file 1: Figure S1. Rarefaction curves across sample types plotted based on OTUs abundance using the R package iNEXT [76] and ggplot2 [77]. Figure S2. NMDS ordination on Bray-Curtis distances plotted based on OTUs abundance of all finch and environmental microbiota from three rearing groups. Figure S3. Cumulative distribution of BrayCurtis dissimilarity calculated pairwise between hatchling gut samples from hand- (solid lines) or finch-reared groups (dashed lines) and other samples. Figure S4. Bubble plot of average relative abundances and IndVal showing the most prevalent bacterial families in each sample type from three rearing groups. Figure S5. Plot LEfSe Results of a) 3-, b) 7-, c) 10-dph hatchling and d) adult gut microbiota from three rearing groups.

Additional file 2: Table S1. Metadata for each library in this study.
}

\section{Abbreviations}

AMOVA: Analysis of molecular variance; ANOVA: Analysis of variance; aZF and aSF: Adult zebra finch and society finch; CZF, CSF, and $\mathrm{CH}$ : Chick (hatchling) raised by zebra finch, society finch, and human; LEfSe: Linear discriminant 
analysis effect size; NMDS: Non-metric multidimensional scaling; OTU: Operational taxonomic unit; SF: Society finch; ZF: Zebra finch

\section{Acknowledgements}

We thank Dr. Mei-Yeh Jade Lu and the NGS High Throughput Genomics Core hosted in the Biodiversity Research Center at Academia Sinica for advice and performing the NGS experiments. We are also grateful to HsiangChing Chen for her assistance in illustrations of amiable finches, and Noah Last for comments on the manuscript. This project was supported by Academia Sinica and the Ministry of Science and Technology of Taiwan.

\section{Authors' contributions}

CYC and DW were involved in developing the study concept and design; CYC, CKC, YYC, and AF conducted the fostering experiments, sample collection, and processing; CYC conducted data acquisition and analysis with technical advice from GTWS, as well as have drafted the manuscript; $\mathrm{CMH}$ and DW contributed to developing conceptual ideas, critical revisions, and improvement of the final manuscript. All authors read and approved the final manuscript.

\section{Funding}

This work was supported by a grant from the Ministry of Science and Technology of Taiwan (MOST 108-2311-B-001-031).

\section{Availability of data and materials}

The datasets generated and analyzed in the current study are available in the Sequence Read Archive (SRA) database at NCBI under BioProject ID PRJNA609776.

\section{Ethics approval}

This study was carried out in accordance with the requirements of the Taiwan Animal Protection Law on Scientific Application of Animals and the Institutional Animal Care and Utilization Committee of the Academia Sinica (Protocol ID: IACUC 13-06-555).

\section{Consent for publication}

Not applicable.

\section{Competing interests}

The authors declare that they have no competing interests.

\section{Author details}

${ }^{1}$ Biodiversity Research Center, Academia Sinica, Taipei 115201, Taiwan. 2Department of Pathology, University of Southern California, Los Angeles, CA 90033, USA. ${ }^{3}$ The IEGG and Animal Biotechnology Center, National Chung Hsing University, Taichung 402204, Taiwan. ${ }^{4}$ Department of Molecular Biology and Human Genetics, Tzu Chi University, Hualien 970301, Taiwan.

\section{Received: 7 May 2020 Accepted: 21 July 2020}

\section{Published online: 11 September 2020}

\section{References}

1. de Goffau MC, Lager S, Sovio U, Gaccioli F, Cook E, Peacock SJ, Parkhill J, Charnock-Jones DS, Smith GC. Human placenta has no microbiome but can contain potential pathogens. Nature. 2019;572:329-34.

2. Ley RE, Lozupone CA, Hamady M, Knight R, Gordon Jl. Worlds within worlds: evolution of the vertebrate gut microbiota. Nat Rev Microbiol. 2008; $6: 776$

3. Lozupone CA, Stombaugh Jl, Gordon JI, Jansson JK, Knight R. Diversity, stability and resilience of the human gut microbiota. Nature. 2012:489:220.

4. Bäckhed F, Ley RE, Sonnenburg JL, Peterson DA, Gordon JI. Host-bacterial mutualism in the human intestine. Science. 2005:307:1915-20.

5. Heijtz RD, Wang S, Anuar F, Qian Y, Björkholm B, Samuelsson A, Hibberd ML, Forssberg $\mathrm{H}$, Pettersson S. Normal gut microbiota modulates brain development and behavior. Proc Natl Acad Sci. 2011;108:3047-52

6. Tremaroli V, Bäckhed F. Functional interactions between the gut microbiota and host metabolism. Nature. 2012:489:242

7. David LA, Maurice CF, Carmody RN, Gootenberg DB, Button JE, Wolfe BE, Ling AV, Devlin AS, Varma Y, Fischbach MA. Diet rapidly and reproducibly alters the human gut microbiome. Nature. 2014;505:559.
8. Dominguez-Bello MG, Costello EK, Contreras M, Magris M, Hidalgo G, Fierer $\mathrm{N}$, Knight R. Delivery mode shapes the acquisition and structure of the initial microbiota across multiple body habitats in newborns. Proc Natl Acad Sci. 2010;107:11971-5.

9. Makki K, Deehan EC, Walter J, Bäckhed F. The impact of dietary fiber on gut microbiota in host health and disease. Cell Host Microbe. 2018;23:705-15.

10. Maslowski KM, Mackay CR. Diet, gut microbiota and immune responses. Nat Immunol. 2011;12:5-9.

11. Rothschild D, Weissbrod O, Barkan E, Kurilshikov A, Korem T, Zeevi D, Costea PI, Godneva A, Kalka IN, Bar N. Environment dominates over host genetics in shaping human gut microbiota. Nature. 2018;555:210.

12. Yatsunenko T, Rey FE, Manary MJ, Trehan I, Dominguez-Bello MG, Contreras M, Magris M, Hidalgo G, Baldassano RN, Anokhin AP. Human gut microbiome viewed across age and geography. nature. 2012;486:222.

13. Zanardo V, Svegliado G, Cavallin F, Giustardi A, Cosmi E, Litta P, Trevisanuto D. Elective cesarean delivery: does it have a negative effect on breastfeeding? Birth. 2010;37:275-9.

14. Benson AK, Kelly SA, Legge R, Ma F, Low SJ, Kim J, Zhang M, Oh PL, Nehrenberg D, Hua K. Individuality in gut microbiota composition is a complex polygenic trait shaped by multiple environmental and host genetic factors. Proc Natl Acad Sci. 2010;107:18933-8.

15. Bevins $C L$, Salzman NH. The potter's wheel: the host's role in sculpting its microbiota. Cell Mol Life Sci. 2011:68:3675.

16. Bolnick DI, Snowberg LK, Hirsch PE, Lauber CL, Org E, Parks B, Lusis AJ, Knight R, Caporaso JG, Svanbäck R. Individual diet has sex-dependent effects on vertebrate gut microbiota. Nat Commun. 2014;5:4500.

17. Moeller AH, Suzuki TA, Phifer-Rixey M, Nachman MW. Transmission modes of the mammalian gut microbiota. Science. 2018:362:453-7.

18. Chen C-Y, Chen P-C, Weng FC-H, Shaw GT-W, Wang D. Habitat and indigenous gut microbes contribute to the plasticity of gut microbiome in oriental river prawn during rapid environmental change. PLoS One. 2017;12: e0181427.

19. Moeller AH, Foerster S, Wilson ML, Pusey AE, Hahn BH, Ochman H. Social behavior shapes the chimpanzee pan-microbiome. Sci Adv. 2016;2: e1500997.

20. Tung J, Barreiro LB, Burns MB, Grenier J-C, Lynch J, Grieneisen LE, Altmann J, Alberts SC, Blekhman R, Archie EA. Social networks predict gut microbiome composition in wild baboons. elife. 2015:4:e05224.

21. Funkhouser $L J$, Bordenstein SR. Mom knows best: the universality of maternal microbial transmission. PLoS Biol. 2013:11:e1001631.

22. Dominguez-Bello MG, De Jesus-Laboy KM, Shen N, Cox LM, Amir A, Gonzalez A, Bokulich NA, Song SJ, Hoashi M, Rivera-Vinas JI. Partial restoration of the microbiota of cesarean-born infants via vaginal microbial transfer. Nat Med. 2016;22:250.

23. Palmer C, Bik EM, DiGiulio DB, Relman DA, Brown PO. Development of the human infant intestinal microbiota. PLoS Biol. 2007;5:e177.

24. Colston TJ, Jackson CR. Microbiome evolution along divergent branches of the vertebrate tree of life: what is known and unknown. Mol Ecol. 2016;25: 3776-800.

25. Arboleya S, Bahrami B, Macfarlane S, Gueimonde M, Macfarlane G, de Los RGC. Production of immune response mediators by HT-29 intestinal cell-lines in the presence of Bifidobacterium-treated infant microbiota. Benefic Microbes. 2015:6:543-52.

26. de Agüero MG, Ganal-Vonarburg SC, Fuhrer T, Rupp S, Uchimura Y, Li H, Steinert A, Heikenwalder M, Hapfelmeier S, Sauer U. The maternal microbiota drives early postnatal innate immune development. Science. 2016;351:1296-302.

27. Gensollen T, lyer SS, Kasper DL, Blumberg RS. How colonization by microbiota in early life shapes the immune system. Science. 2016;352:539-44.

28. Round $J L$, Mazmanian SK. The gut microbiota shapes intestinal immune responses during health and disease. Nat Rev Immunol. 2009;9:313.

29. Grond K, Lanctot RB, Jumpponen A, Sandercock BK. Recruitment and establishment of the gut microbiome in arctic shorebirds. FEMS Microbiol Ecol. 2017:93:fix142.

30. Martín-Vivaldi M, Soler JJ, Peralta-Sánchez JM, Arco L, Martín-Platero AM Martínez-Bueno M, Ruiz-Rodríguez M, Valdivia E. Special structures of hoopoe eggshells enhance the adhesion of symbiont-carrying uropygial secretion that increase hatching success. J Anim Ecol. 2014:83:1289-301.

31. van Veelen HPJ, Salles JF, Tieleman BI. Microbiome assembly of avian eggshells and their potential as transgenerational carriers of maternal microbiota. ISME J. 2018;12:1375. 
32. Ding J, Dai R, Yang L, He C, Xu K, Liu S, Zhao W, Xiao L, Luo L, Zhang Y. Inheritance and establishment of gut microbiota in chickens. Front Microbiol. 2017:8:1967.

33. Ehrlich P, Dobkin DS, Wheye D. Birder's handbook: Simon and Schuster; 1988

34. van Veelen HPJ, Salles JF, Tieleman BI. Multi-level comparisons of cloacal, skin, feather and nest-associated microbiota suggest considerable influence of horizontal acquisition on the microbiota assembly of sympatric woodlarks and skylarks. Microbiome. 2017;5:156.

35. Ruiz-Rodriguez M, Lucas FS, Heeb P, Soler JJ. Differences in intestinal microbiota between avian brood parasites and their hosts. Biol J Linn Soc. 2009;96:406-14

36. Ruiz-Rodríguez M, Martín-Vivaldi M, Martínez-Bueno M, Soler J. Gut microbiota of great spotted cuckoo nestlings is a mixture of those of their foster magpie siblings and of cuckoo adults. Genes. 2018;9:381.

37. Hird SM, Carstens BC, Cardiff SW, Dittmann DL, Brumfield RT. Sampling locality is more detectable than taxonomy or ecology in the gut microbiota of the brood-parasitic brown-headed cowbird (Molothrus ater). PeerJ. 2014;2:e321.

38. Lucas FS, Heeb P. Environmental factors shape cloacal bacterial assemblages in great tit Parus major and blue tit P. caeruleus nestlings. J Avian Biol. 2005; 36:510-6.

39. Teyssier A, Lens L, Matthysen E, White J. Dynamics of gut microbiota diversity during the early development of an avian host: evidence from a cross-foster experiment. Front Microbiol. 2018;9:1524.

40. Mak SS, Wrabel A, Nagai H, Ladher RK, Sheng G. Zebra finch as a developmental model. genesis. 2015;53:669-77.

41. Olson CR, Wirthlin M, Lovell PV, Mello CV. Proper care, husbandry, and breeding guidelines for the zebra finch, Taeniopygia guttata. Cold Spring Harb Protoc. 2014;2014:pdb. prot084780.

42. Shaw RC, Feeney WE, Hauber ME. Nest destruction elicits indiscriminate con-versus heterospecific brood parasitism in a captive bird. Ecol Evol. 2014; 4:4500-4.

43. Engelbrektson A, Kunin V, Wrighton KC, Zvenigorodsky N, Chen F, Ochman $H$, Hugenholtz P. Experimental factors affecting PCR-based estimates of microbial species richness and evenness. ISME J. 2010;4:642-7.

44. Hamady M, Walker JJ, Harris JK, Gold NJ, Knight R. Error-correcting barcoded primers for pyrosequencing hundreds of samples in multiplex. Nat Methods. 2008;5:235-7.

45. Illumina I. 16S Metagenomic sequencing library preparation. Preparing $16 \mathrm{~S}$ Ribosomal RNA Gene Amplicons for the Illumina MiSeq System; 2013. p. 1-28.

46. Magoč T, Salzberg SL. FLASH: fast length adjustment of short reads to improve genome assemblies. Bioinformatics. 2011;27:2957-63.

47. Schloss PD, Westcott SL, Ryabin T, Hall JR, Hartmann M, Hollister EB, Lesniewski RA, Oakley BB, Parks DH, Robinson CJ. Introducing mothur: open-source, platform-independent, community-supported software for describing and comparing microbial communities. Appl Environ Microbiol. 2009;75:7537-41.

48. Edgar RC, Haas BJ, Clemente JC, Quince C, Knight R. UCHIME improves sensitivity and speed of chimera detection. Bioinformatics. 2011;27:2194200.

49. Edgar RC. UPARSE: highly accurate OTU sequences from microbial amplicon reads. Nat Methods. 2013;10:996.

50. DeSantis TZ, Hugenholtz P, Larsen N, Rojas M, Brodie EL, Keller K, Huber T, Dalevi D, Hu P, Andersen GL. Greengenes, a chimera-checked 16S rRNA gene database and workbench compatible with ARB. Appl Environ Microbiol. 2006:72:5069-72.

51. Hothorn T, Bretz F, Westfall P, Heiberger RM, Schuetzenmeister A, Scheibe S, Hothorn MT. Package 'multcomp'. Vienna: Simultaneous inference in general parametric models Project for Statistical Computing; 2016.

52. Lozupone C, Knight R. UniFrac: a new phylogenetic method for comparing microbial communities. Appl Environ Microbiol. 2005;71:8228-35.

53. Clarke KR. Non-parametric multivariate analyses of changes in community structure. Aust J Ecol. 1993;18:117-43.

54. Oksanen J, Blanchet FG, Kindt R, Legendre P, Minchin PR, O'hara R, Simpson GL, Solymos P, Stevens MHH, Wagner H. Package 'vegan'. Community ecology package, version. 2013;2:1-295.

55. Excoffier L, Smouse PE, Quattro JM. Analysis of molecular variance inferred from metric distances among DNA haplotypes: application to human mitochondrial DNA restriction data. Genetics. 1992;131:479-91.

56. Knights D, Kuczynski J, Charlson ES, Zaneveld J, Mozer MC, Collman RG, Bushman FD, Knight R, Kelley ST. Bayesian community-wide cultureindependent microbial source tracking. Nat Methods. 2011;8:761.
57. Waite DW, Taylor MW. Characterizing the avian gut microbiota: membership, driving influences, and potential function. Front Microbiol. 2014;5:223.

58. Segata N, Izard J, Waldron L, Gevers D, Miropolsky L, Garrett WS, Huttenhower C. Metagenomic biomarker discovery and explanation. Genome Biol. 2011;12:R60.

59. Videvall E, Song SJ, Bensch HM, Strandh M, Engelbrecht A, Serfontein N, Hellgren O, Olivier A, Cloete S, Knight R. Major shifts in gut microbiota during development and its relationship to growth in ostriches. Mol Ecol. 2019.

60. Sprockett D, Fukami T, Relman DA. Role of priority effects in the early-life assembly of the gut microbiota. Nat Rev Gastroenterol Hepatol. 2018;15: 197-205.

61. Barbosa A, Balagué V, Valera F, Martínez A, Benzal J, Motas M, Diaz J, Mira A, Pedrós-Alió C. Age-related differences in the gastrointestinal microbiota of chinstrap penguins (Pygoscelis antarctica). PLoS One. 2016;11:e0153215.

62. van Dongen WF, White J, Brandl HB, Moodley Y, Merkling T, Leclaire S, Blanchard P, Danchin É, Hatch SA, Wagner RH. Age-related differences in the cloacal microbiota of a wild bird species. BMC Ecol. 2013;13:11.

63. Lee MD, Newell DG. Campylobacter in poultry: filling an ecological niche. Avian Dis. 2006:50:1-9.

64. Bull SA, Thomas A, Humphrey T, Ellis-Iversen J, Cook AJ, Lovell R, Jorgensen F. Flock health indicators and Campylobacter spp. in commercial housed broilers reared in Great Britain. Appl Environ Microbiol. 2008;74:5408-13.

65. Oakley BB, Lillehoj HS, Kogut MH, Kim WK, Maurer JJ, Pedroso A, Lee MD, Collett SR, Johnson TJ, Cox NA. The chicken gastrointestinal microbiome. FEMS Microbiol Lett. 2014;360:100-12.

66. Benskin $\mathrm{CMH}$, Rhodes G, Pickup RW, Wilson K, Hartley IR. Diversity and temporal stability of bacterial communities in a model passerine bird, the zebra finch. Mol Ecol. 2010;19:5531-44.

67. Olnood CG, Beski SS, Choct M, lji PA. Novel probiotics: their effects on growth performance, gut development, microbial community and activity of broiler chickens. Animal Nutr. 2015:1:184-91.

68. Quigley EM. Prebiotics and probiotics; modifying and mining the microbiota. Pharmacol Res. 2010;61:213-8.

69. van der Wielen P, Keuzenkamp D, Lipman LV, Knapen F, Biesterveld S. Spatial and temporal variation of the intestinal bacterial community in commercially raised broiler chickens during growth. Microb Ecol. 2002;44: 286-93.

70. Yin Y, Lei F, Zhu L, Li S, Wu Z, Zhang R, Gao GF, Zhu B, Wang X. Exposure of different bacterial inocula to newborn chicken affects gut microbiota development and ileum gene expression. ISME J. 2010;4:367.

71. Carmody RN, Gerber GK, Luevano JM Jr, Gatti DM, Somes L, Svenson KL, Turnbaugh PJ. Diet dominates host genotype in shaping the murine gut microbiota. Cell Host Microbe. 2015;17:72-84.

72. Zmora N, Suez J, Elinav E. You are what you eat: diet, health and the gut microbiota. Nat Rev Gastroenterol Hepatol. 2019;16:35-56.

73. Ambrosini R, Corti M, Franzetti A, Caprioli M, Rubolini D, Motta VM, Costanzo A, Saino N, Gandolfi I. Cloacal microbiomes and ecology of individual barn swallows. FEMS Microbiol Ecol. 2019;95:fiz061.

74. Gillingham MAF, Béchet A, Cézilly F, Wilhelm K, Rendón-Martos M, Borghesi F, Nissardi S, Baccetti N, Azafzaf H, Menke S. Offspring microbiomes differ across breeding sites in a panmictic species. Front Microbiol. 2019;10:35.

75. Goodenough AE, Stallwood B, Dandy S, Nicholson TE, Stubbs H, Coker DG. Like mother like nest: similarity in microbial communities of adult female pied flycatchers and their nests. J Ornithol. 2017;158:233-44.

76. Hsieh T, Ma K, Chao A. iNEXT: an R package for rarefaction and extrapolation of species diversity (H ill numbers). Methods Ecol Evol. 2016;7: 1451-6.

77. Wickham H, Chang W, Wickham MH. Package 'ggplot2'. Create Elegant Data Visualisations Using the Grammar of Graphics Version. 2016:2:1-189.

\section{Publisher's Note}

Springer Nature remains neutral with regard to jurisdictional claims in published maps and institutional affiliations. 